\title{
Late neurological complications due to laminar hook compression in idiopathic scoliosis surgery
}

\author{
Albert Ferrando ${ }^{1}$ - Paloma Bas ${ }^{1}$ - Teresa Bas ${ }^{1}$ \\ Received: 28 July 2017 / Accepted: 9 September 2017 \\ (C) International Spinal Cord Society 2017
}

\begin{abstract}
Introduction To provide a review of unusual neurological complications occurring years after spinal instrumentation surgery.

Case Presentation The authors analyzed the cases of three patients with adolescent idiopathic scoliosis who presented with delayed neurological symptoms (later than 12 months postoperatively) following spinal deformity surgery using Texas Scottish Rite Hospital (TSRH) hook-rod instrumentation. One case presented with conus medullaris syndrome while the last two were rare cases of posterior cord syndrome due to a dislodged laminar hook at the thoracolumbar transition.

Discussion In all three cases, the neurological symptoms were due to direct laminar hook compression. Metallosis was observed extra-spinally around the hook-rod construct but not at the sublaminar site. The dislodged laminar hook responsible for the symptoms was situated at the concavity of the curve with the blade-tip pointing downwards. Symptomatic improvement was observed in all patients following complete hardware removal. Conscientious long-term follow-up of patients undergoing posterior spinal fusion (PSF) for adolescent idiopathic scoliosis (AIS) is important in order to recognize late neurological deficits directly related to instrumentation. Anamnesis and physical examination are key for initial assessment and diagnosis, while myelography and computed tomography (CT) images are of great help to confirm the site of spinal compression. Removal of the dislodged laminar hooks after PSF with TSRH instrumentation in AIS cases can potentially improve neurological symptoms.
\end{abstract}

\section{Introduction}

Late neurological complications, occurring years after successful spinal instrumentation, have rarely been reported in the literature [1-15]. Although some authors suggest that neurological symptoms appear due to the formation of epidural metallosis adjoining the hook-rod construct, the cause of the new-onset pain in our cases was related to direct compression of the canal by dislodged laminar hooks in the thoracolumbar region $[1,11]$. This review comprises one report on a case of conus medullaris syndrome and two case reports on posterior cord syndromes resulting from dislodgement of laminar hooks occurring years after posterior spinal fusion and Texas Scottish Rite Hospital

Teresa Bas

teresabas@gmail.com

1 Spine Surgery Unit, Hospital Universitari i Politècnic La Fe, Valencia 46026, Spain
(TSRH) rod instrumentation for adolescent idiopathic scoliosis.

\section{Case Presentation}

\section{Case 1}

A 29-year-old man with a 6-month history of difficulty in walking was seen at the outpatient clinic of our hospital for sudden severe low-back pain, bowel and bladder dysfunction, and lower limb weakness. Four years earlier, in 1990, he had been operated on for thoracolumbar scoliosis through posterior correction and T4-L4 arthrodesis using TSRH hook-rod instrumentation (Fig. 1). The patient presented with no history of trauma.

Upon examination, the patient presented with ataxic gait. He had diminished light touch and pinprick sensation in the lower extremities as well as saddle (S3-S5) anesthesia. Symmetric muscle weakness was observed in the lower 


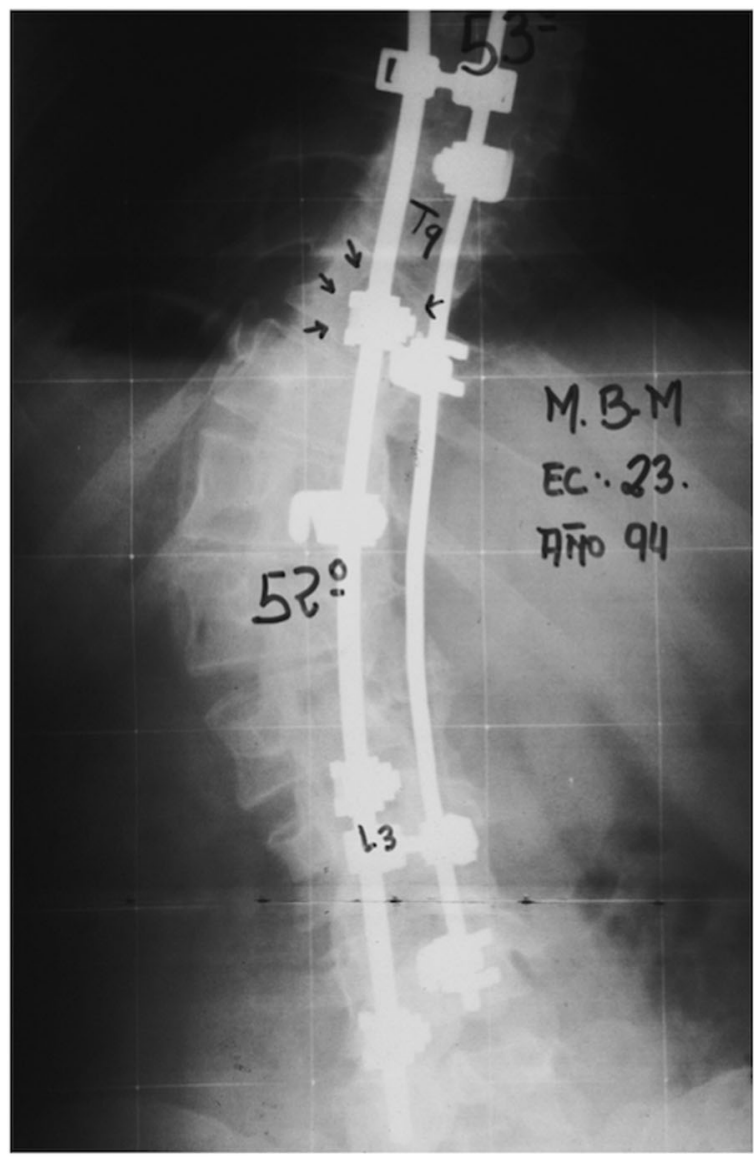

Fig. 1 Patient 1. At 4 years post-op from posterior correction, instrumentation, and T4-L4 fusion, medial migration of the T10 hook can be observed

extremities as well as decreased strength during hip flexion (4/5), knee extension (4/5), ankle dorsiflexion (3/5), and plantar flexion (3/5). The patient noticed more intense left side than right side radicular pain on physical examination maneuvers. Hyperactivity of the deep tendon reflexes and decreased muscle tone were also noted. Sacral segments showed preserved (bulbocavernosus and anal wink) reflexes. Signs and symptoms were consistent with a diagnosis of conus medullaris syndrome (mixed upper and lower motor neuron signs) and patient was graded as ASIA class D.

Radiographs indicated possible migration of the implants, and the myelography demonstrated filling defects in the contrast column at T10 and T12. Following the myelogram, a computed tomography (CT) scan revealed protrusion of the T10 and T12 laminar hooks into the spinal canal (Fig. 2).

A revision procedure carried out that same year (1994) made it possible to find and document corrosion fatigue in the instrumented area T10-T12 16]. Macroscopic findings of metallosis were most evident around the hook-rod junction T10-T12 11]. Although no purulent material was

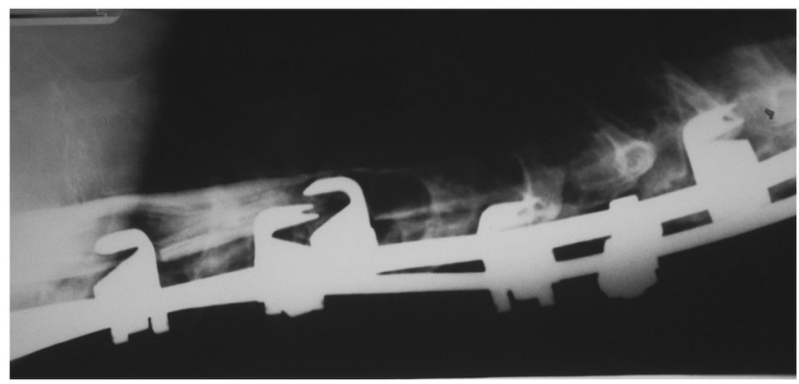

Fig. 2 Patient 1. Myelography demonstrating a tapered column of contrast medium and filling of defect at T10 and T12

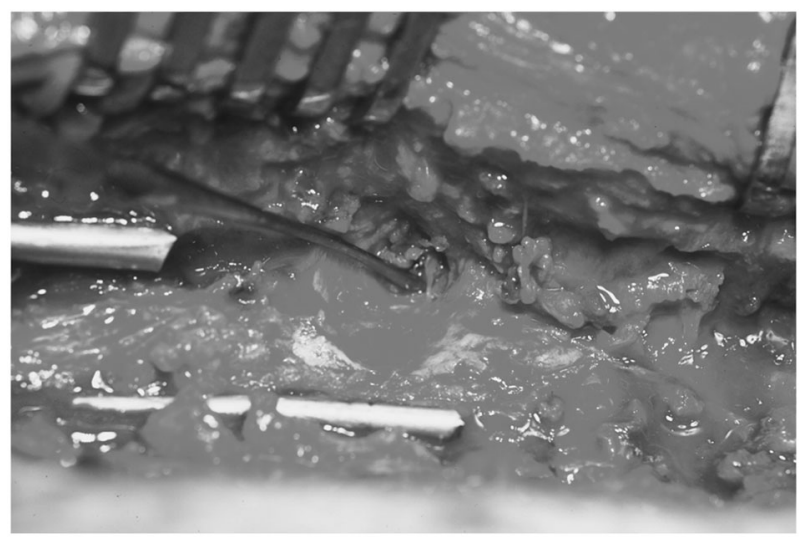

Fig. 3 Intraoperatively, osteolysis caused by dislodged laminar hook at T10 with absence of intraspinal metallosis

seen, a black film was observed surrounding the hook-rod construct, particularly at the thoracolumbar transition. The spinal implant was not broken or disassembled 10]. In addition, the L3 hook had extruded from the lamina. Complete removal of the instrumentation together with the surrounding extraspinal debris was carried out (Fig. 3).

At 1 year follow-up, the patient presented with nearly complete recovery of his motor weakness and sensory deficits. However, spasticity had increased. In 2016, a follow-up examination in the outpatient clinic confirmed that the patient was pain-free, with complete motor and sensory recovery and without bowel or bladder dysfunction. A recent posteroanterior (PA) standing spinal plain film showed no further progression of the scoliotic deformity (Fig. 4).

\section{Case 2}

A 21-year-old man was admitted to the outpatient clinic of our hospital in March 1999 due to a 2-year history of back pain, worsening gait difficulties, and clumsiness. Five years earlier, in January 1994, he had undergone surgery in another hospital for AIS correction. The operation had comprised posterior correction and stabilization using a 


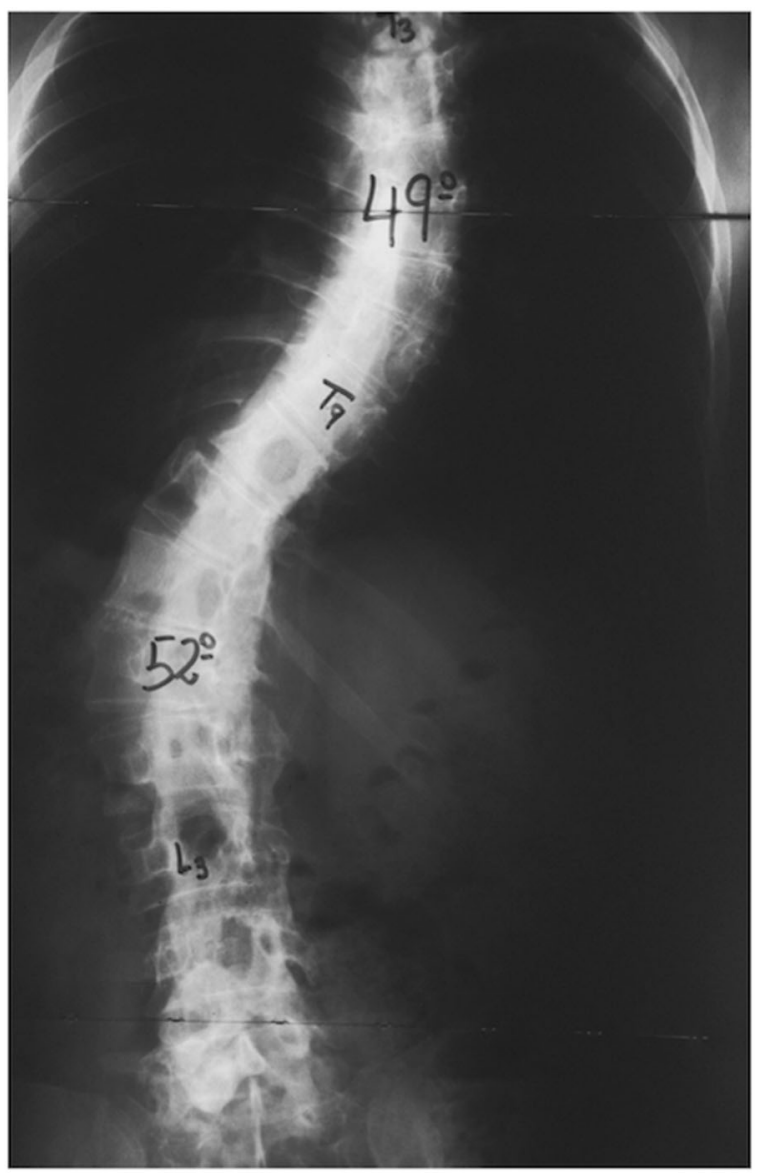

Fig. 4 Postoperative view following surgery. Evidence of osteolysis at site of previously dislodged T10, T12, and L3 hooks

single TSRH hook-rod construct from T5 to T12. The patient referred no history of trauma or changes in bowel or bladder function.

Upon physical examination, the patient presented sensory ataxia. He adopted a wide base, taking slow steps under visual guidance, with the sole of the foot stomping the floor and making a loud noise 17]. Romberg test was positive. The patient was asked to stand up with his feet together and both hands resting on his hips, which he accomplished without difficulty. However, when asked to close his eyes, he was unable to maintain balance. A cranial nerve exam and an upper extremity neurological exam showed normal results. Rectal tone, perianal sensation, and lower extremity motor function and sensitivity were all normal. Lower extremity deep tendon reflexes demonstrated hyperreflexia.

Motor conduction studies of the peroneal and tibial nerves, and sensory conduction studies of the sural and superficial peroneal nerves were normal. F-wave responses of the tibial and peroneal nerves, and the H-reflex of the soleus muscle were also normal. In addition, transcranial brain stimulation revealed normal central conduction time,

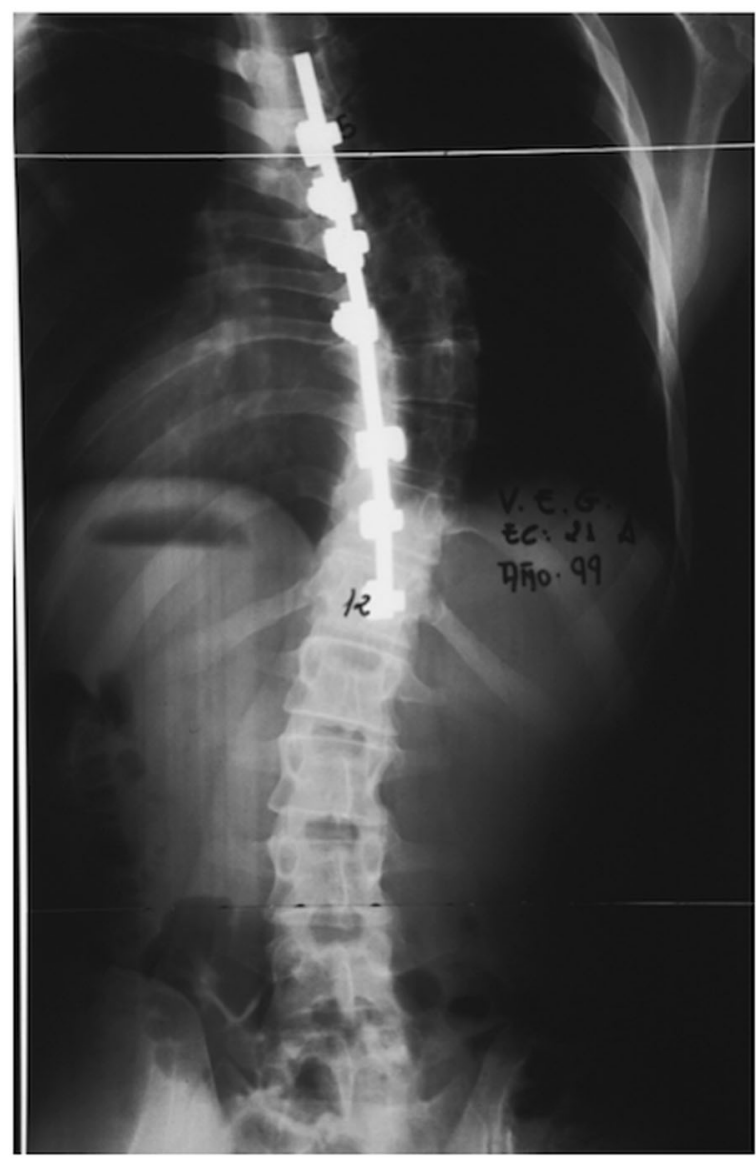

Fig. 5 Patient 2. Postoperative PA radiograph when first symptoms appeared, showing evident medial migration of distal hook-rod construct

amplitude, and MEP/CMAP ratio, indicating integrity of the corticospinal tract.

Anteroposterior (AP) and lateral X-ray spinal images of the spine were obtained (Fig. 5). Myelography was supplemented by CT scanning, which revealed spinal cord compression by laminar hooks at T10, T11, and T12.

Consequently, surgery was performed to remove the TSRH implants. No evidence of metalloma was observed intraoperatively; only scar tissue was identified and removed. The authors found that the distal portion of the hook-rod construct had migrated medially. Additional osseous decompression of intact dorsal spine elements was not indicated as the myelogram had detected isolated compression by the laminar hooks 10]. Within 6 weeks post-op, the patient progressed from his previous gait pattern to a normal gait. At 10 years' follow-up, the patient was painfree without any symptoms.

\section{Case 3}

In February 2014, a 40-year-old man with a 2-year history of chronic back pain and difficulty in walking was 


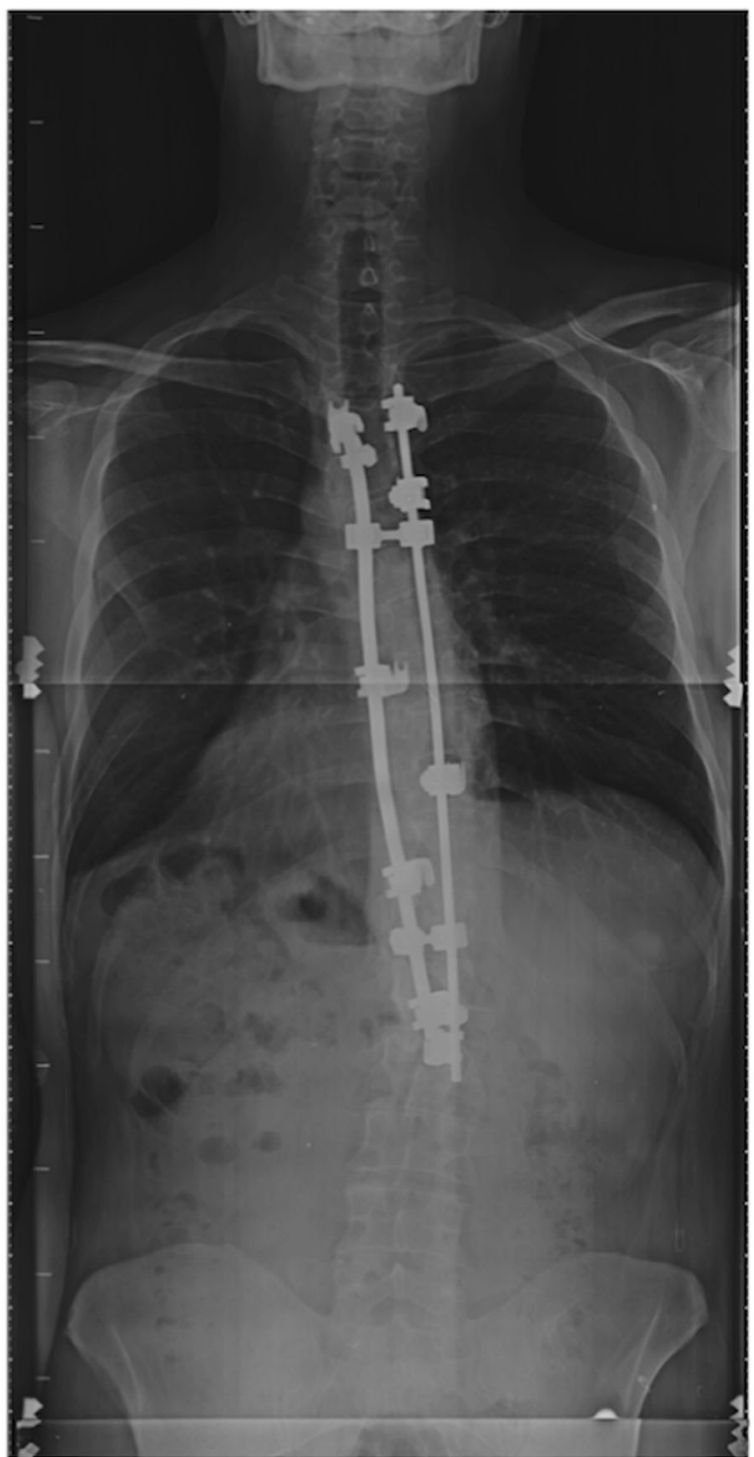

Fig. 6 Patient 3. Standing PA film demonstrating medial migration of distal left hook-rod instrumentation

transferred from his regional hospital to the Neurophysiology Department of our center for performance of electrical studies of the central nervous system and peripheral nerves. In 1990, he had undergone scoliosis surgery with subsequent fusion and TSRH instrumentation from T4 to L2. (Fig. 6).

At the regional hospital, motor conduction studies and Fwave studies of the deep peroneal nerve had been performed, together with sensory conduction studies of the sural nerve, all of which appeared to be normal bilaterally. A needle EMG of L2-S1 was also normal bilaterally.

At our hospital, the neurophysiology team used transcranial magnetic stimulation to investigate nervous propagation along the corticospinal tract, spinal roots, and peripheral nerves 18]. The results were compatible with a possible injury of the posterior column of the spine due to

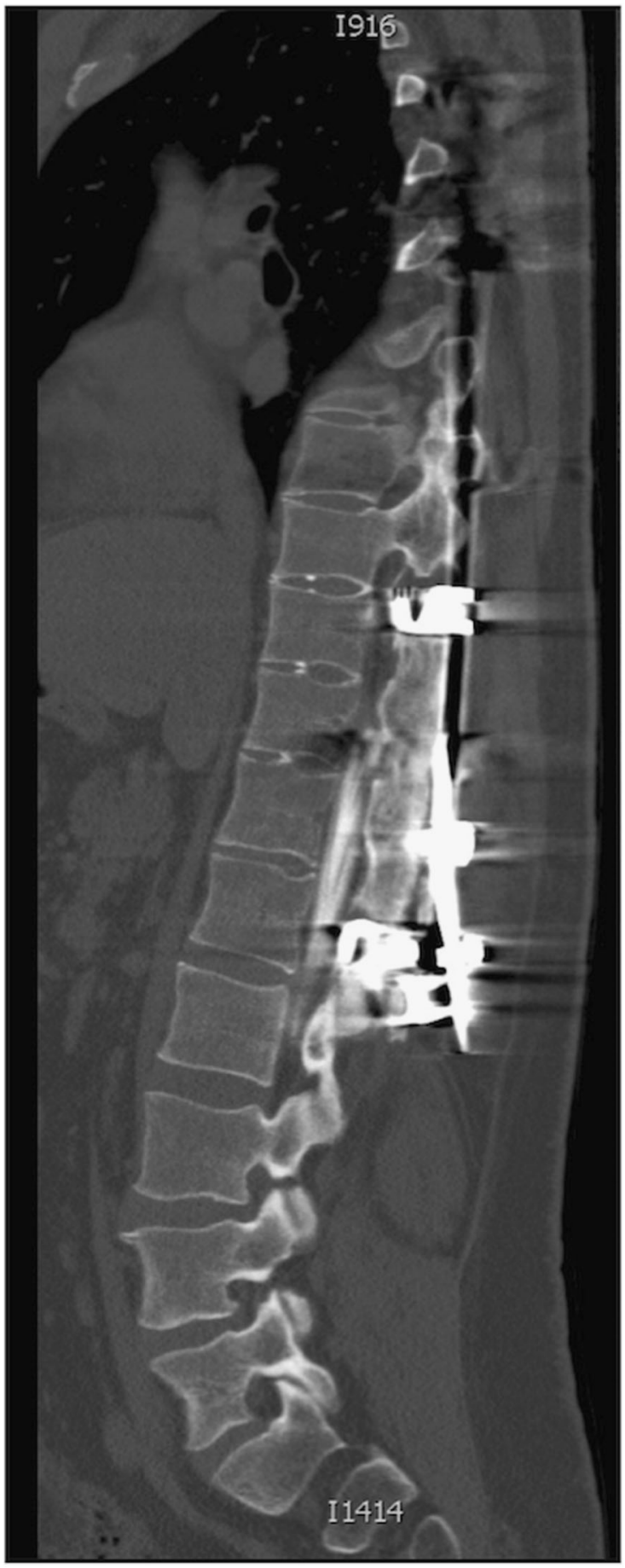

Fig. 7 Patient 3. CT myelography showing the tapered column of contrast medium next to the laminar hook and distal hook-rod construct loosening

involvement of motor pathways. The Spine Surgery Department was consulted for assistance in evaluation and management.

Upon physical examination, the patient showed sensory ataxic or "stomping" gait, relying completely on visual input. He presented with a positive Romberg test and loss of 


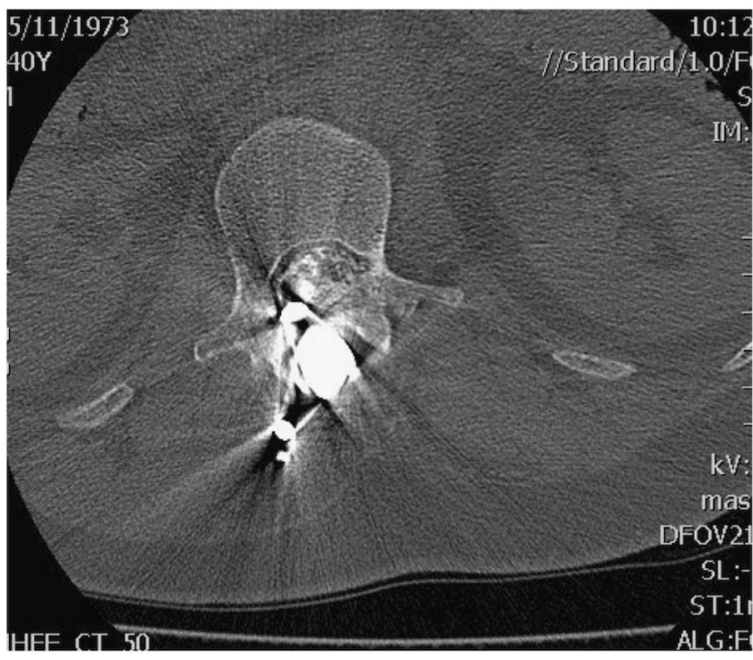

Fig. 8 Patient 3. CT imaging: medial migration of the T11 laminar hook into the spinal canal

two-point discrimination, vibration, and conscious proprioception at T12 and below. Deep tendon reflexes were normal and symmetrical $(1+)$ in the upper extremity (biceps, brachioradialis, and triceps) and $(2+)$ in lower extremity (patellar, Achilles). Muscle strength was normal and symmetrical (5/5) in all major muscle groups of the upper and lower extremity. In addition, the patient had normal bowel and bladder function.

CT scanning with myelography showed focal indentation on the column of contrast medium at the level of the T11 laminar hook (Fig. 7). The other hooks were correctly placed with no evidence of compression at any other level (Fig. 8).

As in the previous case, symptoms were compatible with posterior cord syndrome. The patient was therefore scheduled for implant removal surgery. During the surgery, the T11 hook was found to have separated from the rod. Macroscopically evident yellow-black material was observed at the hook-rod junction extending into the surrounding fibrous tissues but not to the sublaminar site. Two specimens of the metallotic mass were sent to the Department of Pathology. Histologic examination revealed granulation tissue characterized by multiple mononuclear and polymorphonuclear inflammatory cells, capillary infiltration and dense matted fibrin deposits. In addition, numerous histiocytes with abundant vacuolated cytoplasm, foreignbody type multinucleated giant cells containing needleshaped metal particles, and hemosiderin-laden macrophages were present.

After surgery, the patient was admitted to the Department of Neurological Rehabilitation of our hospital and began an intensive balance, gait, and posture re-education program. Two years post-discharge, the patient's gait improved significantly but was not yet completely normal. Plain films demonstrated no progression of the scoliotic deformity.

\section{Discussion}

This study presents 3 cases of late neurological complications; two unique cases of posterior cord syndrome and one case of conus medullaris syndrome due to laminar hook compression. The incidence of neural complications due to spinal deformity surgery has been reported to range between 0.26 and $17 \%[13,19]$. However, most studies refer to immediate postoperative complications $(<6$-month followup) rather than to long-term complications (occurring years after scoliosis surgery) such as those presented here. Moreover, literature on late neurological complications consists of case reports that make no mention of posterior cord syndrome resulting from direct laminar hook compression following spinal instrumentation. In fact, acute posterior cord syndrome has often been mentioned as a rare occurrence mostly related to vascular compromise; only in one case was it reported in association with a posttraumatic hyperextension injury 20].

In all three cases, neurological symptoms can be explained by fretting corrosion of the modular TSRH spinal implant in the thoracolumbar region, where dislodged laminar hooks caused compression of the spinal cord [16, 21, 22]. This was confirmed both by CT myelography and intraoperatively, where the laminar hooks were seen to have come loose causing a iatrogenic injury to the spine.

It is important to note that, in all cases, hook dislodgments were distal and placed on the concavity of the curve. As reported by Vereijken et al. [12], the major intraoperative corrective force occurs at the concavity of the curve where translation of the apex of the curve is toward the midline. Therefore, apical concave anchor points are pulled laterally (in these right convex cases, away from the spinal canal) while proximal and distal anchor points are pulled medially toward the spinal canal. In our series, as pedicle screws were not used distally (since 2000 the authors have used all pedicle screw constructs for AIS), the distal laminar hook(s) migrated medially. The reason why the distal anchor points on the concave side are pulled medially more often than the proximal anchor points, lies in the increased amount of stress that distal anchor points are submitted to in the course of the activities of daily living. It must be said that the thoracolumbar transition and the lumbar spine have a greater range of motion than the thoracic spine, increasing the risk of loosening and fretting of the modular hook-rod construct 5].

Another important aspect in our series was that the laminar hook responsible for the symptoms always had the blade-tip pointing downwards. Since this is a small sample size, it is difficult to establish a conclusion from this observation. However, the authors believe that a downwardpointing blade-tip, if dislodged, is more prone to damaging the spinal cord ("digging-effect") than an upward-pointing 
tip during flexion-extension of the column (flexion pulls the blade-tip away from the column if the blade-tip is pointing upwards). Furthermore, spinal injuries caused by hooks can be understood in terms of volumetric intrusion. Wide-blade TSRH hooks with a downward-pointing blade-tip are responsible for direct compression because they have a greater potential for canal intrusion than pedicle hooks 23].

In addition, no metallotic granuloma was found intraoperatively inside the spinal canal; the fibrous mass was outside, adjacent to the hook-rod construct. All sample tissues analyzed by the Pathology Department yielded similar results to those described in the literature [7-9, 11, 15, 24]. Infection was ruled out as the cause of implant failure as cultures were negative, laboratory infection indexes were normal, and signs and symptoms were absent in all patients 4]. Therefore, the authors believe that distal TSRH hook-rod implant failure due to fretting and corrosion at the thoracolumbar transition results in excessive motion of laminar hooks, producing osteolysis, edema, medial migration, and direct mechanical dural impingement.

Lastly, it is important to note that occurrence of symptoms appears to vary from one patient to another and usually depends on the size and shape of the spinal canal, implant wear and corrosion, and degree of residual mobility in the patient's spinal column 11]. Thus, implant failure must be detected on the basis of abnormalities observed in physical exams carried out years after successful scoliosis instrumentation was performed and in imaging studies ordered early enough to prevent delays in diagnosis.

\section{Compliance with ethical standards}

Conflict of interest The authors declare that they have no competing interests.

\section{References}

1 Beguiristain J, del Rio J, Duart J, Barroso J, Silva A, Villas C. Corrosion and late infection causing delayed paraparesis after spinal instrumentation. J Pediatr Orthop B. 2006;15:320-3.

2 Eismont FJ, Simeone FA. Bone overgrowth (hypertrophy) as a cause of late paraparesis after scoliosis fusion. A case report. J Bone Joint Surg Am. 1981;63:1016-9.

3 Grisafi FN, Emery SE. Migration of laminar hook causing thoracic myelopathy eight years post scoliosis surgery: a case report. Spine (Phila Pa 1976). 2010;35:E228-30.

4 Hales DD, Dawson EG, Delamarter R. Late neurological complications of Harrington-rod instrumentation. J Bone Joint Surg Am. 1989;71:1053-7.

5 Helenius I, Remes V, Yrjonen T, Ylikoski M, Schlenzka D, Helenius M, et al. Harrington and Cotrel-Dubousset instrumentation in adolescent idiopathic scoliosis. Long-term functional and radiographic outcomes. J Bone Joint Surg Am. 2003;85-A:2303-9

6 Kornberg M, Herndon WA, Rechtine GR. Lumbar nerve root compression at the site of hook insertion. Late complication of
Harrington rod instrumentation for scoliosis. Spine (Phila $\mathrm{Pa}$ 1976). 1985; 10:853-5.

7 Obeid I, Vital J-M, Aurouer N, Hansen S, Gangnet N, Pointillart $\mathrm{V}$, et al. Intraspinal canal rod migration causing late-onset paraparesis 8 years after scoliosis surgery. Eur spine J. 2016;25:2097-101

8 Pascal-Moussellard H, Ferrero E, Dubousset J, Miladi L. Expert's comment concerning Grand Rounds case entitled 'Intraspinal canal rod migration causing late-onset paraparesis 8 years after scoliosis surgery' (I. Obeid et al. Eur Spine J; 2014, DOI 10.1007/s00586014-3367-y). Eur spine J. 2016;25:2102-7.

9 Richman SH, Razzano AJ, Morscher MA, Riley PMS. Metallosis presenting as a progressive neurologic deficit four years after a posterior spinal fusion for adolescent idiopathic scoliosis: a case report. Spine (Phila Pa 1976). 2017;42:E56-9.

10 Rittmeister M, Leyendecker K, Kurth A, Schmitt E. Cauda equina compression due to a laminar hook: A late complication of posterior instrumentation in scoliosis surgery. Eur spine $\mathrm{J}$. 1999;8:417-20.

11 Takahashi S, Delecrin J, Passuti N. Intraspinal metallosis causing delayed neurologic symptoms after spinal instrumentation surgery. Spine (Phila Pa 1976). 2001;26:1495-8; discussion 1499

12 Vereijken IMP, de Kleuver M. Late proximal pedicle hook migration into spinal canal after posterior correction surgery of scoliosis causing neurologic deficit: 'proximal junctional scoliosis'? case series and a review of the literature. Spine Deform. 2013;1:229-36.

13 Wilber RG, Thompson GH, Shaffer JW, Brown RH, Nash CLJ. Postoperative neurological deficits in segmental spinal instrumentation. A study using spinal cord monitoring. J Bone Joint Surg Am. 1984;66:1178-87.

14 Botolin S, Merritt C, Erickson M. Aseptic loosening of pedicle screw as a result of metal wear debris in a pediatric patient. Spine (Phila Pa 1976). 2013;38:E38-42.

15 Tezer M, Kuzgun U, Hamzaoglu A, Ozturk C, Kabukcuoglu F, Sirvanci M. Intraspinal metalloma resulting in late paraparesis. Arch Orthop Trauma Surg. 2005;125:417-21.

16 Prikryl M, Srivastava SC, Viviani GR, Ives MB, Purdy GR. Role of corrosion in Harrington and Luque rods failure. Biomaterials. 1989;10:109-17.

17 Bradley W, Thompson P. Gait Disorders. Neurology in Clinical Practice. 4th edn. Philadelphia, PA: Butterworth-Heinemann; 2004.

18 Rossini PM, Rossi S. Transcranial magnetic stimulation: diagnostic, therapeutic, and research potential. Neurology. 2007;68:484-8.

19 Diab M, Smith AR, Kuklo TR. Neural complications in the surgical treatment of adolescent idiopathic scoliosis. Spine (Phila Pa 1976). 2007;32:2759-63.

20 Belen JG, Weingarden SI. Posterior central cord syndrome following a hyperextension injury: case report. Paraplegia. 1988;26:209-11.

21 Soultanis KC, Pyrovolou N, Zahos KA, Karaliotas GI, Lenti A, Liveris I, et al. Late postoperative infection following spinal instrumentation: stainless steel versus titanium implants. J Surg Orthop Adv. 2008;17:193-9

22 Kirkpatrick JS, Venugopalan R, Beck P, Lemons J. Corrosion on spinal implants. J Spinal Disord Tech. 2005;18:247-51.

23 Polly DWJ, Potter BK, Kuklo T, Young S, Johnson C, Klemme WR. Volumetric spinal canal intrusion: a comparison between thoracic pedicle screws and thoracic hooks. Spine (Phila Pa 1976). 2004;29:63-69.

24 Goldenberg Y, Tee JW, Salinas-La Rosa CM, Murphy M. Spinal metallosis: a systematic review. Eur spine J. 2016;25:1467-73. 